\title{
Feasibility of superior vena cava isolation in patients with cardiac implantable electronic devices
}

\author{
Shohei Kataoka ${ }^{1}$, Koichiro Ejima ${ }^{1}$, Kyoichiro Yazaki $^{1}$, Miwa Kanai ${ }^{1}$, Daigo Yagishita ${ }^{1}$, \\ Morio Shoda ${ }^{1}$, and Nobuhisa Hagiwara ${ }^{1}$ \\ ${ }^{1}$ Tokyo Women's Medical University
}

July 16, 2020

\begin{abstract}
Introduction: Some patients with cardiac implantable electronic devices (CIEDs) require atrial fibrillation (AF) ablation, and the superior vena cava (SVC) has been identified as one of the most common non-pulmonary vein foci of AF. This study aimed to investigate the interaction between SVC isolation (SVCI) and CIED leads implanted through the SVC. Methods and Results: We studied 34 patients with CIEDs who had undergone SVCI as part of AF ablation (CIED group), involving a total of 71 CIED leads. A similar number of age-, sex-, and AF type-matched patients without CIEDs formed a control group (non-CIED group). Patients' background and procedural characteristics were compared between the groups. In the CIED group, lead parameters before and after AF ablation were compared, and lead failure after AF ablation was also examined in detail. Procedural characteristics other than fluoroscopic time were similar in both groups. The success rate of SVCI after the final ablation procedure was $91.2 \%$ in the CIED group and $100 \%$ in the non-CIED group; however, these differences were not statistically significant. Lead parameters before and after the AF ablation did not significantly differ between the 2 groups. Lead failure was observed in 3 patients, with a sensing noise in 1 patient and an impedance increase in 2 patients after SVCI. Conclusions: SVCI was achievable without lead failure and significant change in lead parameters in most patients with CIEDs; however, the $8.8 \%$ incidence of lead failure observed after SVCI should be noted.
\end{abstract}

\section{Study design and population}

This study retrospectively analyzed data obtained from the AF ablation database of Tokyo Women's Medical University. From January 2011 to March 2020, 1340 patients had undergone AF ablation at our institution. We excluded 266 patients who did not undergo SVCI. Thirty-four patients who had received CIED implantation before SVCI were enrolled in this study (CIED group), and 34 age-, sex- and AF type-matched patients without CIEDs comprised a control group (non-CIED group). Experienced physicians undertook the data collection, based on individual chart reviews. The following data were used as baseline characteristics: age, sex, height, body weight, AF type, structural heart diseases, echocardiographic parameters, and B-type natriuretic peptide levels. Procedural characteristics were also assessed. Baseline patient and procedural characteristics were compared between the CIED and non-CIED groups. In the CIED group, detailed device-related and lead-related information was studied, along with lead parameters before and after SVCI. Lead positions were also evaluated using computed tomography imaging before AF ablation at the level of the left atrium roof, and these were divided into 4 portions, namely, the anterior, septal, posterior, and lateral portions of the SVC.

The study protocol conformed to the ethical guidelines of the 1975 Declaration of Helsinki and was approved by the Institutional Review Board and Ethics Committee of the Tokyo Women's Medical University. Written informed consent was obtained from all patients.

Ablation procedure 
Details of the AF ablation procedure performed at our institution have previously been described. ${ }^{13}$ Therefore, we present a brief description focusing on SVCI as follows. All CIEDs were interrogated prior to AF ablation, and bradycardia parameters were programed to VVI 30. In the defibrillator devices, tachyarrhythmia detection and therapy were switched off. All patients had undergone a wide circumferential PVI (CPVI) using a 3-dimensional mapping system (CARTO 3, Biosense Webster, Inc., Diamond Bar, CA) and a 3.5-mm open-irrigated tipped catheter (Navistar ThermoCool, ThermoCool SF, or ThermoCool STSF, Biosense Webster, Inc., Diamond Bar, CA). After the CPVI, an empirical SVCI was performed except in patients without electrical potentials $10 \mathrm{~mm}$ above the right atrium (RA)-SVC junction. Radiofrequency (RF) application was delivered below the circular mapping catheter placed 5-10 $\mathrm{mm}$ above the RA-SVC junction in a point-by-point fashion using a power of $25 \mathrm{~W}$ for $<30$ seconds or $30 \mathrm{~W}$ for $<20$ seconds. Before the RF application, high output pacing at $10 \mathrm{~mA}$ was performed on the anterolateral and posterolateral walls of the SVC. Ablation at the site, where phrenic nerve stimulation was observed, was avoided. If the RA-SVC conduction remained at the site of phrenic nerve stimulation, the RF was delivered carefully while the diaphragm movement on fluoroscopy was monitored using a power of $20 \mathrm{~W}$, for up to 20 seconds. The endpoint of the SVCI was the elimination of SVC potentials recorded using the circular mapping catheter. The absence of dormant conduction with adenosine-triphosphate infusion was also confirmed. After AF ablation, all devices were tested again and reprogramed to baseline settings. A plain chest radiograph was taken to check the lead dislodgement on the day following AF ablation. All patients were followed up at the outpatient clinic at 1, 3, 6, 9, and 12 months after the ablation, then every 6 months thereafter. Recurrence was defined as documented atrial tachyarrhythmias (ATAs) on the electrocardiogram, 24-hour ambulatory monitoring, portable electrocardiographic monitoring, or data obtained from CIEDs (lasting $>30$ seconds). A second AF ablation session was attempted for recurrence of ATAs based on the physicians' discretion.

\section{Outcome measures}

First, we evaluated the success rate of SVCI, comparing the CIED group with the non-CIED group. Other procedural characteristics such as procedural time, time needed to achieve SVCI, fluoroscopic time, the reconnection rate of SVCI in the second session, and the incidence of phrenic nerve paralysis were also compared between the groups. Second, lead parameters were compared before and after SVCI in the CIED group. Lead parameters obtained within 1 year before and after AF ablation were evaluated. Lead parameters after AF ablation were defined as sensing, pacing threshold, and pacing impedance at the outpatient clinic after discharge, to exclude transient abnormality of CIEDs in the acute phase. In patients who had undergone 2 SVCI sessions, we compared lead parameters before the first session and after the second session. Finally, we studied the lead failure rate after SVCI and the details of the lead failure. Lead failure was defined as a sudden impedance increase ( $>50 \%$ compared with chronic values) in pacing and high voltage impedance, lead dislocation, and electrical noise artifact from rapid, non-physiological make-break potentials recorded on the sensing channel. Oversensing of non-cardiac potentials, not considered as lead integrity failure, was not defined as lead failure in this study. ${ }^{14}$

\section{Statistical analysis}

Categorical variables are expressed as numbers and proportions and were compared using Fisher's exact test. Continuous variables are shown as mean and standard deviation or median with interquartile range and were compared using a Student t-test or a Mann-Whitney U test. Lead parameters before and after AF ablation were compared using a paired t-test or a Wilcoxon signed-rank test, as appropriate. We performed 1:1 propensity score-matching analysis based on age, sex, and AF type to compare the results of SVCI and procedural characteristics in the CIED group with those in the non-CIED group. Two-sided $\mathrm{P}<0.05$ was considered statistically significant, and JMP pro13® software (SAS Institute Inc., Cary, NC, USA) was used.

\section{Results}

Comparison of patient and procedural characteristics 
The CIED group comprised 34 patients (mean age, $57.9 \pm 12.7$ years; males, $64.7 \%$ ), and 71 leads, including 44 pacing leads, 21 defibrillator leads, and 6 coronary vein leads, were assessed in this study. Details of patient and procedural characteristics are shown in Table 1. No significant differences in age, sex, AF type, and type of ablation catheters were found. Paroxysmal AF was the most dominant type of AF in both groups. The prevalence of structural heart diseases was significantly higher in the CIED group. The entire procedural time and the time needed to achieve SVCI were similar in both groups; however, the fluoroscopic time was significantly longer in the CIED group (15.8 \pm 1.9 vs. $10.5 \pm 1.8 \mathrm{~min}, \mathrm{P}=0.046)$. No phrenic nerve paralysis due to SVCI was observed in both groups. The success rate of the SVCI in the first session was 97.1\% (33 of 34 patients) in the CIED group. A recurrence of ATAs was observed in 12 (35.3\%) patients of the CIED group and in $10(29.4 \%)$ patients of the non-CIED group, and all these patients had undergone a second session of AF ablation. The rates of SVCI reconnection and PVI reconnection in the second session did not significantly differ between the groups. In the second session for the CIED group, SVCI reconnection was observed in 9 of 12 patients (75.0\%). The reconnection site corresponded with lead positions in all 9 patients. In 2 of 9 patients, SVCI could not be achieved in the second session. As a result, the successful rate of SVCI after the final ablation procedure was $91.2 \%$ in the CIED group and $100 \%$ in the non-CIED group, showing no statistically significant difference $(\mathrm{P}=0.077)$. SVCI failed in 3 patients, and details of these 3 patients are described as follows. In the first AF ablation session, SVCI could not be achieved in only 1 patient. An atrial lead and a dual-coil defibrillator lead were implanted in this patient (Figure 1A). The ablation catheter used for this patient was a 3.5-mm open-irrigated tipped catheter without contact forcesensing technology (Navistar ThermoCool SF, Biosense Webster, Inc., Diamond Bar, CA). In the remaining 2 patients, electrical re-isolation of the SVC failed in the second session. One patient was implanted with 3 leads, including a 2.2- $\mathrm{mm}$ atrial pacing lead, 2.2- $\mathrm{mm}$ ventricular pacing lead, and an abandoned lead. A 3.5-mm open-irrigated tipped catheter with contact force sensing and surround flow technology (ThermoCool STSF, Biosense Webster, Inc., Diamond Bar, CA) was used for this patient. A 2.4-mm atrial lead and a $2.25-\mathrm{mm}$ ventricular lead were implanted into the other patient. The ablation catheter used for this patient was a 3.5-mm open-irrigated tipped catheter without contact force-sensing technology (Navistar ThermoCool SF, Biosense Webster, Inc., Diamond Bar, CA).

\section{Device and lead characteristics}

Detailed information regarding CIEDs is summarized in Table 2. Implantation of PM, ICD, and CRT devices had been performed prior to AF ablation in 13 patients, 15 patients, and 6 patients, respectively. The median period from the first generator implantation to the first AF ablation was 71.5 months (interquartile range, 31.3-129.8 months). A total of 73 leads, including abandoned leads, were implanted in all study patients, but data and parameters regarding the abandoned leads were not obtained. Consequently, this study evaluated 71 leads, as described above. The number of leads implanted through the SVC comprised 1 lead in 3 patients, 2 leads in 23 patients, and 3 leads in 8 patients, as well as 2 abandoned leads. Detailed lead characteristics such as lead diameter and outer insulation materials are shown in Table 2. Of 71 leads, the leads were most frequently positioned at the lateral portion of the SVC (76.1\%), followed by the posterior portion of the SVC (16.9\%). The leads were positioned at the septal portion of the SVC in only 1 patient with a CIED implanted on the right.

\section{Lead parameters before and after SVCI}

Table 3 shows a comparison of lead parameters before and after the SVCI. Lead impedance of pacing leads, defibrillator leads, shocking coil, and CV leads did not significantly differ before and after the SVCI. No significant differences were found in pacing thresholds and sensing in the pacing leads, defibrillator leads, and coronary vein leads.

\section{Details of adverse effects of AF ablation on CIEDs}

In this study, no permanent pulse generator malfunctions and no dislodgement of leads were observed. However, lead failure was observed in 3 of 34 patients, with a sensing noise in 1 patient and a significant impedance increase in 2 patients after SVCI. A 67-year-old man showed sensing noises 2 months after the 
second SVCI. He had undergone implantation of a dual chamber pacemaker 16 years before the first AF ablation. No sensing noises were observed after the first SVCI. The second session of the AF ablation was performed 14 months after the first session. Electrical isolation of the SVC was successfully achieved through $\mathrm{RF}$ application (maximum power, $25 \mathrm{~W}$ ) to the lateral wall of the SVC contiguous to the leads using a 3.5-mm open-irrigated tipped catheter without contact force-sensing technology (Navistar ThermoCool SF, Biosense Webster, Inc., Diamond Bar, CA). Thereafter, sensing noise on an atrial pacing lead was observed. The diameter of the lead body (Stelid BJ45D, ELA Medical, Montrouge, France) was $2.5 \mathrm{~mm}$, and its outer insulation material was silicone. Two patients showed an increase in lead impedance. One was an impedance increase of the ventricular lead (389-795 ohms) implanted 10 years before the ablation. The diameter of the ventricular lead (Thinline II Sterox EZ, Intermedics, TX, USA) was $1.7 \mathrm{~mm}$, and its outer insulation material was polyurethane. The other was an impedance increase of the atrial pacing lead (456-779 ohms) implanted 4 years before the ablation. The diameter of the atrial lead (CapSure Fix Novus, Medtronic, MN, USA) was $2 \mathrm{~mm}$, and its outer insulation was silicone. Both leads functioned normally; therefore, an additional procedure such as lead exchange was not required.

\section{Discussion}

This study investigated whether leads in the SVC might become obstacles in achieving electrical isolation of the SVC and whether RF application during SVCI might damage the leads. The main findings of this study were as follows: (i) SVCI was successfully achieved in $91.2 \%$ of the study patients after the final ablation procedure, (ii) lead parameters did not significantly differ after SVCI; however, (iii) 3 lead failures $(8.8 \%$ of the study patients) were observed after SVCI.

Feasibility of SVCI in patients with CIEDs

Several previous reports have shown the histological and electrophysiological characteristics of the SVC. It has been microscopically confirmed by autopsy of human hearts that the myocardial sleeve of the SVC only extends approximately $1.5 \mathrm{~cm}$ from the $\mathrm{RA}^{15}$; however, many previous reports have demonstrated arrhythmogenicity of the SVC. ${ }^{16-18}$ In contrast, the electrical potential of the SVC has been recorded approximately $4 \mathrm{~cm}$ above the RA-SVC junction. ${ }^{19}$ Because breakthrough points from the RA to the SVC are limited, circumferential RF application is not always needed to achieve SVCI. ${ }^{20}$ Therefore, RF applications to the site where the leads had been positioned could be avoided in most cases. Recently, conduction patterns between the SVC and the RA have been studied using ultra-high-resolution mapping. A spontaneous conduction block line was observed in $50 \%$ of the study patients, and the block line was mainly positioned from the lower lateral wall to the higher anterior wall or from the lower posterolateral wall to the higher mid-septal wall. ${ }^{19} \mathrm{We}$ confirmed that most of the leads passed through the lateral portion of the SVC. This might explain why most of the leads did not become obstacles for SVCI, which resulted in the high success rate for SVCI in the CIED group. Electrical reconnection between the SVC and the RA was observed in 9 of 12 patients who underwent the second session among the CIED group. The reconnection sites corresponded with the SVC wall contiguous to leads in all patients, which might have resulted from insufficient RF application at the site closest to the CIED leads. SVCI failed in 3 patients ( $8.8 \%$ of the study patients) after the final ablation procedure, which indicated that the target myocardial sleeve might have been in direct contact with CIED leads in these 3 patients. One patient in whom SVCI failed in the first ablation session had a dual-coil defibrillator lead. The SVC coil was located at the lateral wall of the SVC with a long adhering lesion, which might result in the electrical isolation failure of the SVC. (Figure 1A) Two patients in whom electrical re-isolation of the SVC failed in the second ablation session had leads with diameters of $>2 \mathrm{~mm}$. Therefore, leads with a large diameter were associated with a large contact area to target the myocardial sleeve of the SVC, which might result in SVCI failure.

\section{Adverse effects of SVCI on CIED leads}

$\mathrm{RF}$ application to the myocardium contiguous to CIED leads is needed to achieve electrical isolation of the SVC in some patients with previously implanted CIEDs; however, the general consensus of the Heart Rhythm Society is that direct contact between ablation catheters and CIED leads should be avoided. ${ }^{21}$ 
Potential interaction between SVCI and CIEDs may include the following: pulse generator malfunction, lead dislodgement during AF ablation, outer insulation damage, change in impedance, pacing, and sensing parameters because of RF application to the site closest to the leads. This study revealed no permanent pulse generator malfunction; however, the effects of RF application on the pulse generator, including noise reversion mode with asynchronous pacing, oversensing of electromagnetic interference, and mode switch or transient reset to elective replacement interval, have been previously reported. ${ }^{22}$ Previous studies have shown atrial lead dislodgement after catheter ablation of AF in patients with CIEDs and a rise in the pacing threshold after atrioventricular node ablation in these patients. ${ }^{23,24} \mathrm{AF}$ ablation carries higher risks of lead dislodgement when performing transseptal puncture or catheter manipulation in the RA, compared with catheter ablation of arrhythmias other than AF. In the present study, impedance rise was observed in 2 patients. The mechanism of a pacing threshold rise remains unclear; however, micro-dislodgement or lead damage due to inductive current might contribute to a pacing threshold rise. The dislodgement of leads is a concern, especially given the current trend for fewer fluoroscopy AF ablation procedures using a 3-dimensional mapping system. Therefore, careful catheter manipulation on fluoroscopic imaging may be important. A previous study involving AF ablation in 86 patients with CIEDs reported atrial lead dislodgement in 2 patients with newly implanted leads of $<6$ months. ${ }^{23}$ Distal tip encapsulation within the myocardium has been reported to occur $2-6$ weeks after implantation of leads. ${ }^{25,26}$ In our study, the median period from CIED implantation to AF ablation was 71.5 months, and the fluoroscopic time was significantly longer in the CIED group than in the control group. As a result, there was no lead dislodgement after SVCI. An adverse effect on lead insulation is another issue in SVCI for patients with CIEDs because the myocardium, when achieving electrical isolation of the SVC, might be close to the CIED leads. Lead insulation damage is one of the most common reasons for defibrillator lead failure. ${ }^{27} \mathrm{~A}$ previous in vitro study evaluated the adverse effect of direct application of RF on pacing and defibrillator lead function. In that study, CIED leads, including all commercially available lead insulation materials, were tested. RF energy was delivered at a maximum power of $50 \mathrm{~W}$, with irrigated and non-irrigated tipped catheters. Significant changes in lead function and outer insulation were not observed. ${ }^{28}$ In another study that assessed the effects of electrocautery on lead insulation materials ${ }^{29}$ lead insulation damage was shown to be more common in polyurethane and copolymer leads. In our study, no significant changes were found in lead parameters such as impedance, pacing threshold, and sensing. Sensing noise was observed in 1 patient, although whether RF application directly influenced lead insulation in that case remains unclear. The outer insulation material of the lead was silicone, which has been reported to be less susceptible to thermal damage from RF application. ${ }^{29}$ Moreover, the reported lead survival rate of the same model lead (Stelid BJ45D, ELA Medical, Montrouge, France) was 100\% at 4.5 years after implantation, and that of ventricular leads (Stelid BT45D, ELA Medical, Montrouge, France) with the same structure, other than the proximal electrode position, was $99 \%$ at 20 years after implantation. Therefore, RF application might damage the outer insulation of the leads, and an association between RF ablations and sensing noises could not be completely ruled out, which is in contrast to the findings in the above-mentioned previous studies. There might be a difference between in vitro and in vivo studies. Micro-cracking resulting from environmental stress and metal-induced oxidation is known as outer insulation malfunction of leads implanted for a long time. Another possible reason for the sensing noises may be the unique structure of the lead. The lead (Stelid BJ45D, ELA Medical, Montrouge, France) had 2 electrodes, and the proximal electrode was positioned $70 \mathrm{~mm}$ from the distal tip (Figure 1B). RF energy might be applied to the proximal electrode when performing SVCI. Therefore, the safety of RF application to the CIED leads has been not fully guaranteed in the clinical setting.

This study had some limitations. First, this was a retrospective, single-center study. Second, the study population who had undergone both SVCI and CIED implantation prior to SVCI was relatively small. Third, the relationship between RF application and lead failure was not determined. The occurrence of sensing noise and an impedance increase might be compatible with lead degradation over time. Finally, the durability of SVCI in patients with CIED was not determined because not all study patients had undergone a second session. 


\section{Conclusion}

SVCI could be achieved without lead failure and significant change of lead parameters in most patients with CIEDs; however, the $8.8 \%$ incidence of lead failure observed after SVCI should be noted. Careful RF application is essential to avoid not only sinus node injury and phrenic nerve damage but also lead damage for patients with CIEDs. In addition, lead structures, outer insulation materials, and the position of the leads or the defibrillator coil in the SVC must be carefully examined prior to SVCI. Further studies with a larger sample size are needed to confirm the safety and efficacy of SVCI for patients with CIEDs in the clinical setting.

\section{References}

1. Terasawa T, Balk EM, Chung M, et al. Systematic review: comparative effectiveness of radiofrequency catheter ablation for atrial fibrillation. Ann Intern Med 2009;151:191-202.

2. Aleksandr V, Jeremy M, Nadav H, et al. Revisiting pulmonary vein isolation alone for persistent atrial fibrillation: a systematic-review and meta-analysis. Heart Rhythm 2017;14:661-667.

3. Lin WS, Tai CT, Hsieh MH, et al. Catheter ablation of paroxysmal atrial fibrillation initiated by nonpulmonary vein ectopy. Circulation 2003;107:3176-3183.

4. Ejima K, Kato K, Iwanami Y, et al. Impact of an empiric isolation of the superior vena cava in addition to circumferential pulmonary vein isolation on the outcome of paroxysmal atrial fibrillation ablation. Am J Cardiol 2015;116:1711-1716.

5. Li JY, Jiang JB, Zhong GQ, Ke HH, He Y. Comparison of empiric isolation and conventional isolation of superior vena cava in addition to pulmonary vein isolation on the outcome of paroxysmal atrial fibrillation ablation. Int Heart J 2017;58:500-505.

6. Zhang T, Wang Y, Liang Z, et al. Effect of combined pulmonary vein and superior vena cava isolation on the outcome of second catheter ablation for paroxysmal atrial fibrillation. Am J Cardiol 2020;125:1845-1850.

7. Akoum N, McGann C, Vergara G, et al. Atrial fibrosis quantified using late gadolinium enhancement MRI is associated with sinus node dysfunction requiring pacemaker implant. J Cardiovasc Electrophysiol 2012;23:44-50.

8. Chang HY, Lin YJ, Lo LW, et al. Sinus node dysfunction in atrial fibrillation patients: the evidence of regional atrial substrate remodelling. Europace 2013;15:205-211.

9. Lamas GA, Lee K, Sweeney M, et al. The mode selection trial (MOST) in sinus node dysfunction: design, rationale, and baseline characteristics of the first 1000 patients. Am Heart J 2000;140:541-551.

10. Prabhu S, Taylor AJ, Costello BT, et al. Catheter ablation versus medical rate control in atrial fibrillation and systolic dysfunction: the CAMERA-MRI study. J Am Coll Cardiol 2017;70:1949-1961.

11. Marrouche NF, Brachmann J, Andresen D, et al. Catheter ablation for atrial fibrillation with heart failure. N Engl J Med 2018;378:417-427.

12. Al-Khatib SM, Stevenson WG, Ackerman MJ, et al. 2017 AHA/ACC/HRS Guideline for Management of Patients with Ventricular Arrhythmias and the Prevention of Sudden Cardiac Death: A Report of the American College of Cardiology/American Heart Association Task Force on Clinical Practice Guidelines and the Heart Rhythm Society. J Am Coll Cardiol 2018;72:e91-e220.

13. Ejima K, Henmi R, Iwanami Y, Yagishita D, Shoda M, Hagiwara N. Comparison of the efficacy of empiric thoracic vein isolation for the treatment of paroxysmal and persistent atrial fibrillation in patients without structural heart disease. J Cardiovasc Electrophysiol 2017;28:266-272. 
14. Rordorf R, Poggio L, Savastano S, et al. Failure of implantable cardioverter-defibrillator leads: a matter of lead size? Heart Rhythm 2013;10:184-190.

15. Kholova I, Kautzner J. Morphology of atrial myocardial extensions into human caval veins: a postmortem study in patients with and without atrial fibrillation. Circulation 2004;110:483-488.

16. Tsai CF, Tai CT, Hsieh MH, et al. Initiation of atrial fibrillation by ectopic beats originating from the superior vena cava: electrophysiological characteristics and results of radiofrequency ablation. Circulation 2000;102:67-74.

17. Enriquez A, Liang JJ, Santangeli P, Marchlinski FE, Riley MP. Focal atrial fibrillation from the superior vena cava. J Atr Fibrillation 2017;9:1593.

18. Chang KC, Lin YC, Chen JY, Chou HT, Hung JS. Electrophysiological characteristics and radiofrequency ablation of focal atrial tachycardia originating from the superior vena cava. Jpn Circ J 2001;65:1034-1040.

19. Tanaka Y, Takahashi A, Takagi T, et al. Novel ablation strategy for isolating the superior vena cava using ultra high-resolution mapping. Circ J 2018;82:2007-2015.

20. Goya M, Ouyang F, Ernst S, Volkmer M, Antz M, Kuck KH. Electroanatomic mapping and catheter ablation of breakthroughs from the right atrium to the superior vena cava in patients with atrial fibrillation. Circulation 2002;106:1317-1320.

21. Crossley GH, Poole JE, Rozner MA, et al. The Heart Rhythm Society (HRS)/American Society of Anesthesiologists (ASA) Expert Consensus Statement on the perioperative management of patients with implantable defibrillators, pacemakers and arrhythmia monitors: facilities and patient management. This document was developed as a joint project with the American Society of Anesthesiologists (ASA), and in collaboration with the American Heart Association (AHA), and the Society of Thoracic Surgeons (STS). Heart Rhythm 2011;8:1114-1154.

22. Darrat YH, Morales GX, Elayi CS. The effects of catheter ablation on permanent pacemakers and implantable cardiac defibrillators. J Innov Card Rhythm Manag 2017;8:2630-2635.

23. Lakkireddy D, Patel D, Ryschon K, et al. Safety and efficacy of radiofrequency energy catheter ablation of atrial fibrillation in patients with pacemakers and implantable cardiac defibrillators. Heart Rhythm 2005;2:1309-1316.

24. Burke MC, Kopp DE, Alberts M, et al. Effect of radiofrequency current on previously implanted pacemaker and defibrillator ventricular lead systems. J Electrocardiol 2001;34 Suppl:143-148.

25. Bardy GH, Hofer B, Johnson G, et al. Implantable transvenous cardioverter-defibrillators. Circulation 1993;87:1152-1168.

26. Furman S, Pannizzo F, Campo I. Comparison of active and passive adhering leads for endocardial pacing. PACE 1979;2:417-427.

27. Kleemann T, Becker T, Doenges K, et al. Annual rate of transvenous defibrillation lead defects in implantable cardioverter-defibrillators over a period of $>10$ years. Circulation 2007;115:2474-2480.

28. Darrat YH, Agarwal A, Morales GX, et al. Radiofrequency and cryo-ablation effect on transvenous pacing and defibrillatory lead integrity: an in vitro study. J Cardiovasc Electrophysiol 2016;27:976-980.

29. Lim KK, Reddy S, Desai S, et al. Effects of electrocautery on transvenous lead insulation materials. J Cardiovasc Electrophysiol 2009;20:429-435.

\section{Figure legends}

\section{Figure 1.}


(A) A Plain chest radiograph of the patient who received an implantable cardioverter defibrillator. A dualcoil defibrillator lead was implanted through the superior vena cava (SVC), and the SVC coil was contiguous to the lateral wall of the SVC. Electrical isolation of the SVC failed in the first ablation session.

(B) A plain chest radiograph of the patient who received a dual-chamber permanent pacemaker. The proximal electrode of the atrial lead (Stelid BJ45D, ELA Medical, Montrouge, France) was positioned $70 \mathrm{~mm}$ from the distal tip, and the proximal electrode corresponded with the level of the SVC isolation (yellow arrow).

\section{Hosted file}

20200716SVC device study Table1,2,3.docx available at https://authorea.com/users/343606/ articles/470231-feasibility-of-superior-vena-cava-isolation-in-patients-with-cardiacimplantable-electronic-devices

\section{Hosted file}

Figure 1SVC DEVICE .pptx available at https://authorea.com/users/343606/articles/470231feasibility-of-superior-vena-cava-isolation-in-patients-with-cardiac-implantableelectronic-devices 